\title{
Effects of Plan Asymmetry in Inelastic Seismic Response of One-Story Systems
}

\author{
By Rakesh K. Goel, ${ }^{1}$ and Anil K. Chopra ${ }^{2}$
}

\begin{abstract}
The effects of plan asymmetry on the earthquake response of onestory systems are identified by comparing the dynamic response of an asymmetricplan system and the corresponding symmetric-plan system for a wide range of system parameters - uncoupled lateral vibration period, uncoupled torsional-to-lateral frequency ratio, stiffness eccentricity, and yield factor. Elastic as well as inelastic systems are studied. For the latter, two values of the strength eccentricity are considered: equal to the stiffness eccentricity and zero; the latter is representative of code-designed buildings. Based on the response results for a wide range of system parameters, this paper identifies how the structural response is affected by plan asymmetry and how these effects differ between elastic and inelastic systems. It is shown that the response of inelastic systems is affected less by plan asymmetry compared to elastic systems. Between the two types of inelastic systems considered, the response of strength-symmetric systems is affected by plan asymmetry generally to a smaller degree compared to systems with equal strength and stiffness eccentricities.
\end{abstract}

\section{INTRODUCTION}

The effects of coupling between lateral and torsional motions on the earthquake response of asymmetric-plan buildings have been the subject of numerous studies. Initially, most studies were concerned with the elastic response of buildings, and the effects of lateral-torsional coupling for such systems are now well established. In recent years, the focus has shifted to inelastic systems in order to obtain results applicable to the design of buildings (Erdik 1975; Irvine and Kountouris 1980; Kan and Chopra 1981; Tso and Sadek 1985; Bozorgnia and Tso 1986; Esteva 1987; Sadek and Tso 1988; Tso and Hongshan 1990). However, as recently demonstrated (Goel and Chopra 1990b), these studies have not always arrived at consistent conclusions because the results of each investigation are restricted to the particular system and the underlying modeling assumptions. Obviously, a more comprehensive investigation is necessary in order to develop a better understanding of the inelastic response of asymmetric-plan systems, resulting in consistent, generally applicable conclusions that can provide the basis for improving torsional provisions in building codes.

This investigation is aimed toward filling this need. The one-story system investigated, although simple, is chosen to satisfy various requirements suggested by our recent work (Goel and Chopra 1990b) to ensure wide applicability of results. The earthquake response of an asymmetric-plan system and the corresponding symmetric-plan system are compared for a wide range of system parameters-uncoupled lateral vibration period, torsional-to-lateral frequency ratio, stiffness eccentricity, and yield factor-with the objective of identifying how structural response is affected by plan asymmetry.

${ }^{1}$ Grad. Student, Dept. of Civ. Engrg., Univ. of California, Berkeley, CA 94720.

${ }^{2}$ Prof., Dept. of Civ. Engrg., Univ. of California, Berkeley, CA. 


\title{
Effects of Plan Asymmetry in Inelastic Seismic Response of One-Story Systems
}

\author{
By Rakesh K. Goel, ${ }^{1}-\cdots-$ \\ and Anil K. Chopra, ${ }^{2}$
}

\begin{abstract}
The effects of plan asymmetry on the earthquake response of onestory systems are identified by comparing the dynamic response of an asymmetricplan system and the corresponding symmetric-plan system for a wide range of system parameters - uncoupled lateral vibration period, uncoupled torsional-to-lateral frequency ratio, stiffness eccentricity, and yield factor. Elastic as well as inelastic systems are studied. For the latter, two values of the strength eccentricity are considered: equal to the stiffness eccentricity and zero; the latter is representative of code-designed buildings. Based on the response results for a wide range of system parameters, this paper identifies how the structural response is affected by plan asymmetry and how these effects differ between elastic and inelastic systems. It is shown that the response of inelastic systems is affected less by plan asymmetry compared to elastic systems. Between the two types of inelastic systems considered, the response of strength-symmetric systems is affected by plan asymmetry generally to a smaller degree compared to systems with equal strength and stiffness eccentricities.
\end{abstract}

\section{INTRODUCTION}

The effects of coupling between lateral and torsional motions on the earthquake response of asymmetric-plan buildings have been the subject of numerous studies. Initially, most studies were concerned with the elastic response of buildings, and the effects of lateral-torsional coupling for such systems are now well established. In recent years, the focus has shifted to inelastic systems in order to obtain results applicable to the design of buildings (Erdik 1975; Irvine and Kountouris 1980; Kan and Chopra 1981; Tso and Sadek 1985; Bozorgnia and Tso 1986; Esteva 1987; Sadek and Tso 1988; Tso and Hongshan 1990). However, as recently demonstrated (Goel and Chopra 1990b), these studies have not always arrived at consistent conclusions because the results of each investigation are restricted to the particular system and the underlying modeling assumptions. Obviously, a more comprehensive investigation is necessary in order to develop a better understanding of the inelastic response of asymmetric-plan systems, resulting in consistent, generally applicable conclusions that can provide the basis for improving torsional provisions in building codes.

This investigation is aimed toward filling this need. The one-story system investigated, although simple, is chosen to satisfy various requirements suggested by our recent work (Goel and Chopra 1990b) to ensure wide applicability of results. The earthquake response of an asymmetric-plan system and the corresponding symmetric-plan system are compared for a wide range of system parameters - uncoupled lateral vibration period, torsional-to-lateral frequency ratio, stiffness eccentricity, and yield factor-with the objective of identifying how structural response is affected by plan asymmetry.

${ }^{1}$ Grad. Student, Dept. of Civ. Engrg., Univ. of California, Berkeley, CA 94720.

${ }^{2}$ Prof., Dept. of Civ. Engrg., Univ. of California, Berkeley, CA. 


\section{System and Ground Motions}

\section{One-Story System}

The system considered is the idealized one-story building shown in Fig. 1 , which includes resisting elements oriented along the direction of ground motion as well as perpendicular to the ground motion. The latter are included to ensure widely applicable results (Goel and Chopra 1990b). Because the system response is not sensitive to the number of elements along the direction of ground motion, two elements are sufficient (Goel and Chopra 1990b). Resisting elements are frames or walls having strength and stiffness in their planes only. The mass, stiffness, and strength properties of the system are symmetrical about the $x$-axis, but not about the $y$-axis. This lack of symmetry is characterized by the stiffness eccentricity $e_{s}$, the distance between the center of mass (CM) and the center of stiffness (CS) (Goel and Chopra 1990a). Since plan asymmetry in most buildings arises from the distribution of stiffness and not of mass, the system chosen is stiffness-eccentric and not mass-eccentric (Goel and Chopra 1990b).

The natural, elastic vibration frequencies, $\omega$ and $\omega_{\theta}$, of the corresponding symmetric-plan system-a system with $e_{s}=0$, but with the mass $m$ of the rigid deck, the lateral stiffness $K_{y}$ of the system along the $y$-direction, and the torsional stiffness $K_{\theta s}$ of the system about the CS the same as in the asymmetric-plan system-are given as

$\omega=\sqrt{\frac{K_{y}}{m}}$

and

$\omega_{\theta}=\sqrt{\frac{K_{\theta s}}{m r^{2}}}$.

where $r=$ the radius of gyration of the deck about the CM. The ratio of the uncoupled torsional and lateral frequencies is defined as

$\Omega_{\theta}=\frac{\omega_{\theta}}{\omega}$

The force-deformation relationship of each resisting element is assumed

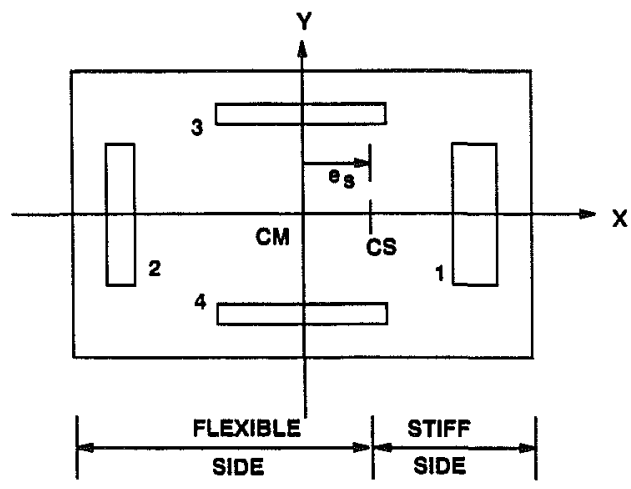

FIG. 1. Idealized One-Story System 
to be elastic-perfectly plastic. The plastic center (or center of strength) is defined as the location of the resultant of yield forces of the resisting elements (Goel and Chopra 1990b; Sadek and Tso 1988). The distance $e_{p}$ between the centers of strength and mass is defined as the strength eccentricity of the system. The strength eccentricity of an asymmetric-plan system is equal to its stiffness eccentricity if the yield forces of its resisting elements are the same as in the corresponding symmetric-plan system (Goel and Chopra 1990a, 1990b). On the other hand, the strength eccentricity is zero if the yield forces of all the resisting elements are the same and if the elements are located symmetrically about the CM. Because the system response is affected by the relative values of the strength eccentricity and stiffness eccentricity (Goel and Chopra 1990b) and the strength eccentricity of codedesigned buildings tends to be much smaller than their stiffness eccentricity (Goel and Chopra 1990a; Tso and Hongshan 1990), both values of $e_{p}$ are considered: $e_{p}=e_{s}$ and $e_{p}=0$.

The yield deformations of resisting elements can be related to the strength eccentricity $e_{p}$ and the yield deformation $u_{y}$ of the corresponding symmetricplan system (Goel and Chopra 1990a). The latter is defined through the dimensionless yield factor $c$ as

$u_{y}=c u_{o}$

where $u_{o}=$ the peak deformation of the corresponding symmetric-plan (SDF) system if it were to remain elastic during the selected ground motion. Thus, $c=1$ implies response within the elastic range for SDF systems but not necessarily for asymmetric-plan systems; $c<1$ indicates yield strength lower than that necessary for the system to remain elastic.

The elastic response of the system of Fig. 1 depends on the following system parameters: uncoupled vibration period $T=2 \pi / \omega$; normalized stiffness eccentricity $e_{s} / r$; ratio of the uncoupled torsional and lateral vibration frequencies $\Omega_{\theta}$; and the damping ratio $\xi$. It has been demonstrated by Goel and Chopra (1990a) that the additional parameters needed to characterize the inelastic response are: the ratio of the uncoupled vibration frequencies in $x$ and $y$-translation $\omega_{x} / \omega$; the ratio $\gamma_{x}$ of the torsional stiffness due to the resisting elements oriented perpendicular to the direction of ground motion to the total torsional stiffness at the CS of the system; the yield coefficient $c$; the value of the strength eccentricity $e_{p}$ relative to the stiffness eccentricity $e_{s}$; and the overstrength factor $O_{s}$, by which the strength of the system exceeds the value if it had no asymmetry in plan. Because the response of code-designed systems, which possess $e_{p}<<e_{s}$, is essentially unaffected by $\gamma_{x}$ and $\omega_{x} / \omega$ (Goel and Chopra 1990a), their values are fixed at typical values of 0.5 and 1 , respectively.

Consequently, the inelastic response of the system considered in this investigation is characterized by the strength eccentricity $e_{p}$ and the yield factor $c$, in addition to all the parameters that characterize the elastic system, i.e., $\omega, \Omega_{\theta}, e_{s} / r$, and $\xi$, note that $O_{s}$ is selected as one.

\section{Ground Motions}

The first ground motion selected is a half-cycle displacement pulse with its displacement, velocity, and acceleration histories shown in Fig. 2. This excitation has the desirable property of a smooth response spectrum (Fig. 2 ), which facilitates identification of the effects of plan asymmetry on structural response. In order to demonstrate that the observations of structural response behavior for the simple input carry over to actual earthquake ground 


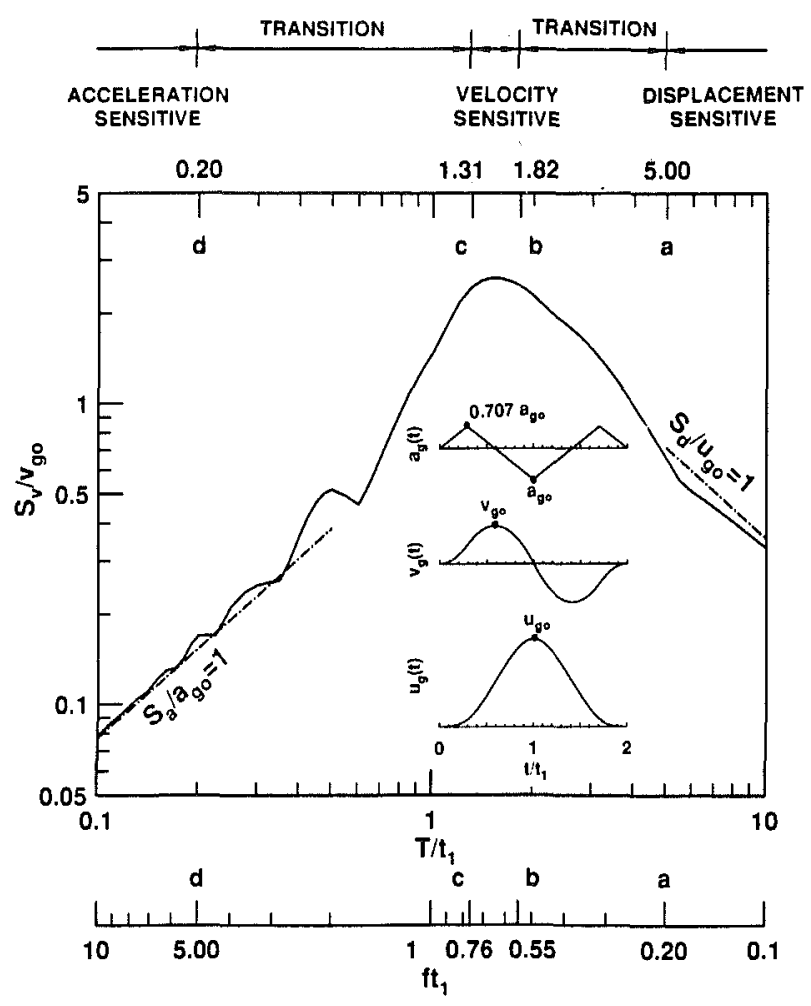

FIG. 2. Response Spectrum for Half-Cycle Displacement Ground Motion with Spectral Regions Identifled

motions, the second ground motion selected is the first $6.3 \mathrm{sec}$ of the SOOE component of the El Centro record obtained during the Imperial Valley earthquake of May 18, 1940 (Fig. 3). Various frequency regions in the response spectra for the two excitations have been identified previously (Veletsos and Vann 1971) and are shown in Figs. 2 and 3, separated by points a, b, c, and $\mathrm{d}$.

\section{Response Quantities}

The earthquake responses of an asymmetric-plan system and the corresponding symmetric-plan system are compared with the objective of identifying how structural response is affected by the coupling of lateral and torsional motions arising from plan asymmetry. For this purpose, the peak lateral displacement $u_{s}$ of the asymmetric-plan system at its CS is compared with the peak lateral deformation $u_{o}$ of the corresponding symmetric-plan system, which is an elastic or inelastic system consistent with the asymmetric-plan system. Therefore, in contrast to Eq. 3, the meaning of $u_{o}$ in the rest of the paper is not restricted to elastic systems. From a design point of view, it would be useful to know how much the design deformation for a resisting element increases because of plan asymmetry. For this purpose $u_{\max }$, the largest of peak deformations among all resisting elements, is also 

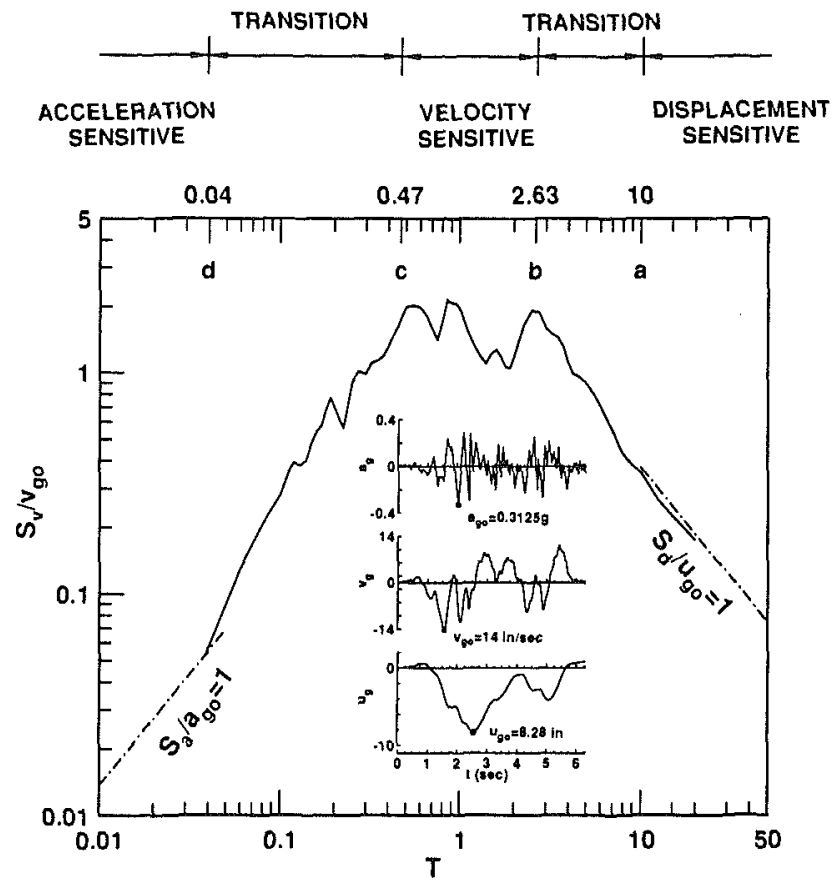

FIG. 3. Response Spectrum for El Centro Ground Motlon with Spectral Regions Identifled

compared with the peak element deformation $u_{o}$ in the corresponding symmetric-plan system.

The torsional response of the one-story asymmetric-plan system may be usefully characterized by the dynamic eccentricity $e_{d}$. Suppose that the ground motion produces peak lateral deformation $u_{s}$, peak base shear $V_{s}$ at the CS, peak torsional deformation $u_{\theta}$, and the peak base torque $T_{\theta s}$ in the asymmetric-plan system. The same excitation causes peak deformation $u_{o}$ and the base shear $V_{o}$ in the corresponding symmetric-plan system. At least two different definitions of $e_{d}$ have been introduced previously for linearly elastic systems: (1) $e_{d}$ is the distance from the CS at which static application of the force $V_{o}$ produces the base torque $T_{\theta s}$ (Hejal and Chopra 1989); and (2) $e_{d}$ is the distance from the CS at which $V_{o}$ should be applied to produce the torsional deformation $u_{\theta}$ (Erdik 1975). The two definitions are conceptually different in that the static and dynamic values of the torque are matched in the first case, in contrast to the torsional deformation in the second case. Needed in this study is a definition for $\boldsymbol{e}_{d}$ that also applies to inelastic systems. In this case, the first definition based on matching of torque is not meaningful because the peak values of torque and base shear are restricted to their yield values. Therefore, the definition based on matching of deformations is selected, i.e.

$e_{d}=\frac{K_{\theta s} u_{\theta}}{V_{o}}=\frac{K_{\theta s} u_{\theta}}{K_{y} u_{o}}$ 
The effects of plan asymmetry, or lateral-torsional coupling, are measured by the deviations of $u_{s} / u_{o}$ and $u_{\max } / u_{o}$ from unity and $e_{d} / r$ from zero. The dynamic amplification of torsional deformation is measured by the increase of $e_{d} / e_{s}$ above unity, which implies that the peak torsional deformation exceeds its value due to static application of the lateral force (or base shear $V_{o}$ ) at a distance $e_{s}$ from the CS. How the effects of plan asymmetry vary with the uncoupled lateral vibration period $T$, the uncoupled torsional-tolateral frequency ratio $\Omega_{\theta}$, and the stiffness eccentricity ratio $e_{s} / r$ is investigated in the subsequent sections.

\section{Influence of Uncoupled Lateral Vibration Period}

The variation of $u_{s} / u_{o}$ and $e_{d} / e_{s}$ against the vibration period $T$ are presented for elastic systems and for two types of inelastic systems: systems with equal strength and stiffness eccentricities $\left(e_{p}=e_{s}\right)$ and strength-symmetric $\left(e_{p}=0\right)$ systems. Such plots are shown in Figs. 4-6 for three values of $e_{s} / r=0.05,0.2$, and 0.5 ; a fixed value of $\Omega_{0}=1$; and for inelastic systems, a single value of $c=0.25$. Similar plots are shown in Figs. 7 and

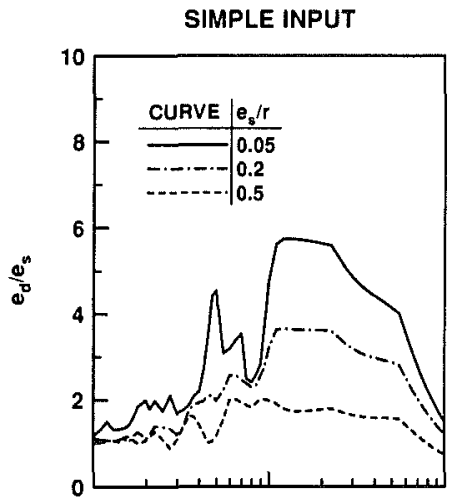

EL CENTRO INPUT
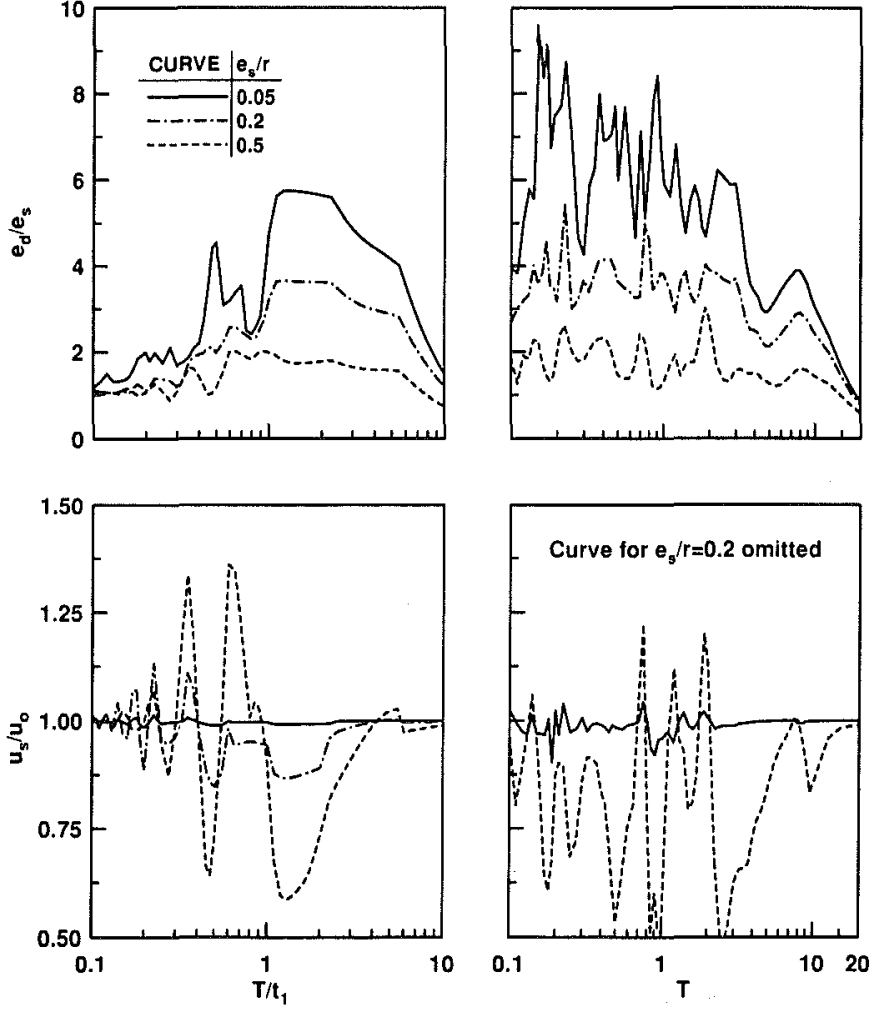

FIG. 4. Ratio of Peak Lateral Deformations of Asymmetric- and Symmetric-Plan Systems $u_{s} / u_{o}$ and Ratio of Dynamic and Static Eccentricities $e_{d} / e_{s}$ for Elastic Systems; $e_{s} / r=0.05,0.2$, and $0.5 ; \Omega_{\theta}=1$, and $\xi=5 \%$ 

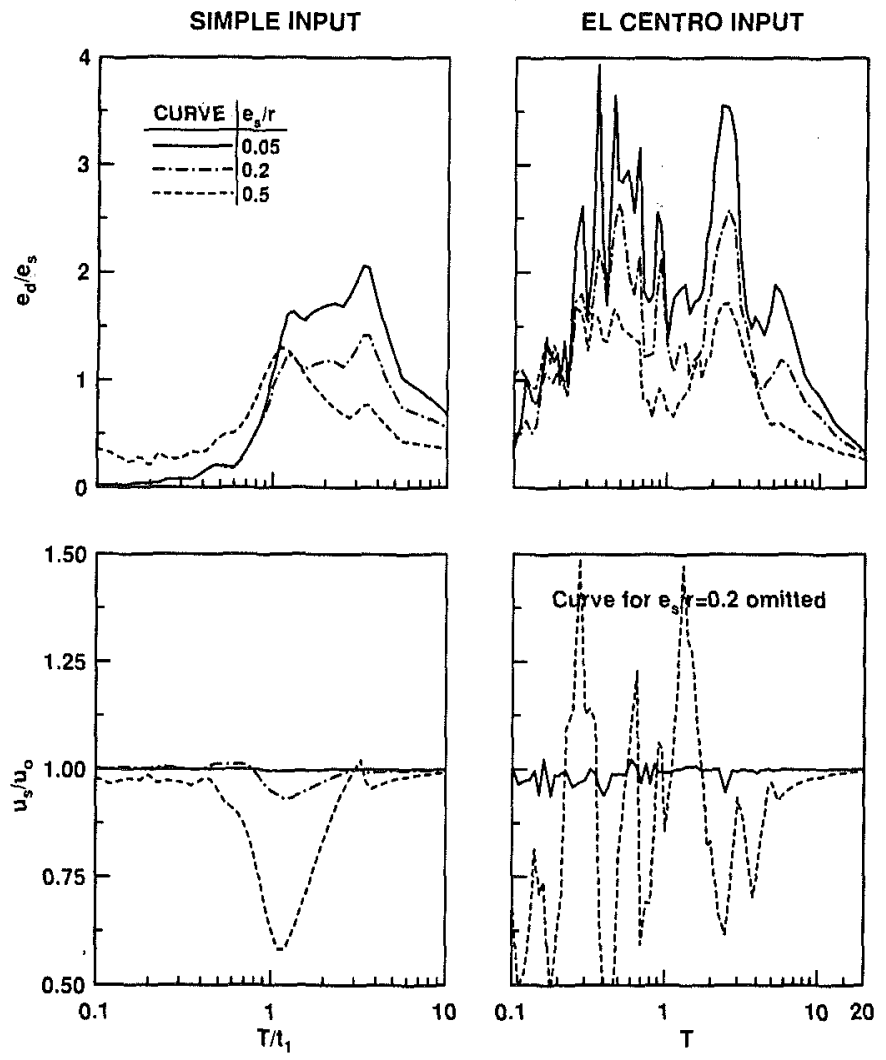

FIG. 5. Ratio of Peak Lateral Deformations of Asymmetric- and Symmetric-Plan Systems $u_{s} / u_{o}$ and Ratio of Dynamic and Static Eccentricities $e_{d} / e_{s}$ for Inelastic Systems with $e_{p}=e_{s}$ and $c=0.25 ; e_{s} / r=0.05,0.2$, and $0.5 ; \Omega_{\theta}=1$, and $\xi=5 \%$

8 for each of the two types of inelastic systems, for two values of the yield factor $c=0.25$ and 0.5 , and compared with elastic systems; $e_{s} / r$ is fixed at 0.2 and $\Omega_{\theta}$ at 1. In Fig. $9, u_{\max } / u_{o}$ is plotted against $T$ for these inelastic systems and compared with elastic systems for a fixed value of $e_{s} / r=0.2$. The frequency ratio is chosen as unity to emphasize the effects of plan asymmetry on the response of elastic systems. System responses to the simple input and El Centro excitation were computed for the same set of system parameters; however, when required for clarity, some of the curves have been omitted from the figures associated with the El Centro excitation.

Plan asymmetry causes torsional deformation, as shown by $e_{d}>0$ in Figs. 4-6, which does not occur in the corresponding symmetric-plan system. It also modifies the lateral deformation $u_{o}$ experienced by the corresponding symmetric-plan system, resulting in a smaller or larger deformation $u_{s}$, depending on the lateral vibration period $T$. In contrast, plan asymmetry was shown to reduce the lateral deformation of a system no matter what its vibration period when the structural response was calculated by response spectrum analysis of elastic systems with the ground motion characterized by 

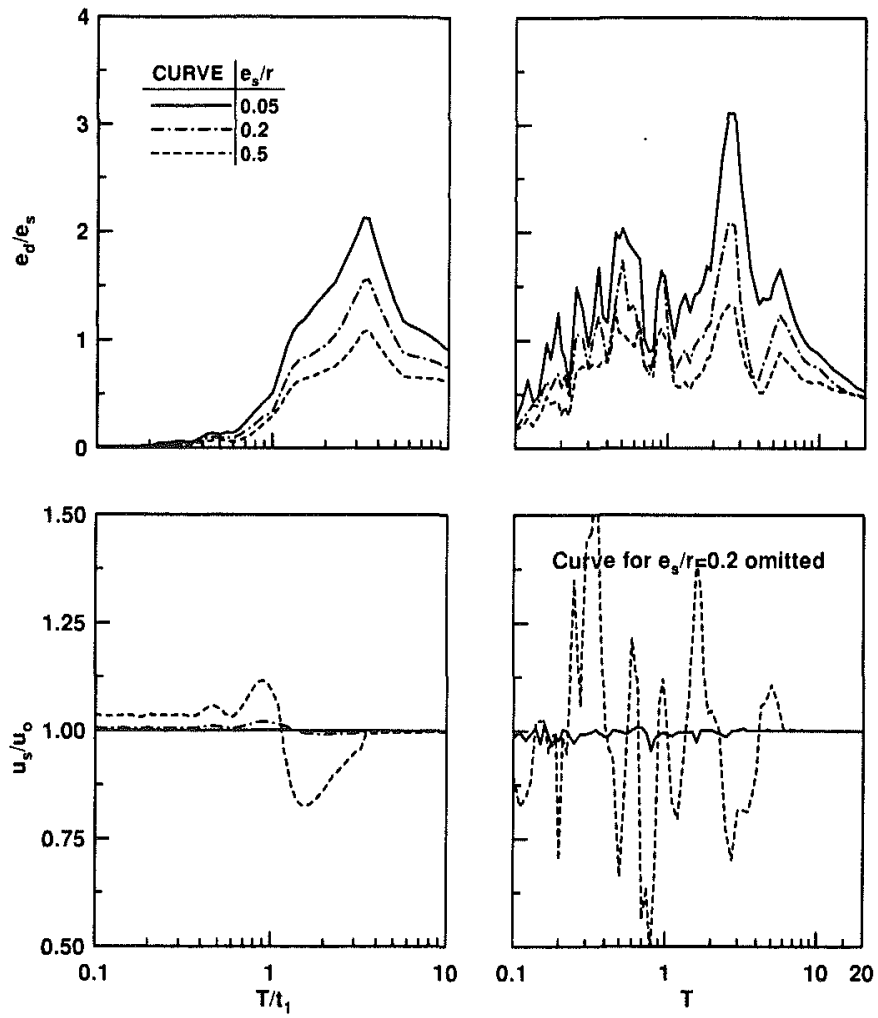

FIG. 6. Ratio of Peak Lateral Deformations of Asymmetric- and Symmetric-Plan Systems $u_{s} / u_{o}$ and Ratio of Dynamic and Static Eccentricities $e_{d} / e_{s}$ for Inelastic Systems with $e_{p}=0$ and $c=0.25 ; e_{s} / r=0.05,0.2$, and $0.5 ; \Omega_{\theta}=1$, and $\xi=5 \%$

smooth spectra (Hejal and Chopra 1989). Thus, it is apparent that the effects of plan asymmetry in the time-history response of elastic systems vary with period $T$, especially for realistic excitations, such as the El Centro ground motion. As $e_{s} / r$ increases, i.e., as the structural plan becomes more asymmetric, the variability of $u_{s} / u_{o}$ for elastic systems with respect to the period $T$ increases, implying that the effects of plan asymmetry become increasingly sensitive to the period $T$. In contrast, the ratio $e_{d} / e_{s}$ is most sensitive to the period for elastic systems with small eccentricity. However, the period dependence of the effects of plan asymmetry tends to be less pronounced for inelastic systems and decreases with increasing inelastic action (decreasing c) (Figs. 7 and 8).

The plan-asymmetry effects are especially significant for medium-period systems in the velocity-sensitive and neighboring transition regions of the spectrum, where these effects are sensitive to the stiffness eccentricity (Figs. 4-6) and to the yield factor (Figs. 7 and 8). The ratio $e_{d} / e_{s}$ of dynamic eccentricity to its static value tends to reach its largest value for mediumperiod, velocity-sensitive systems. This dynamic amplification of torsional 

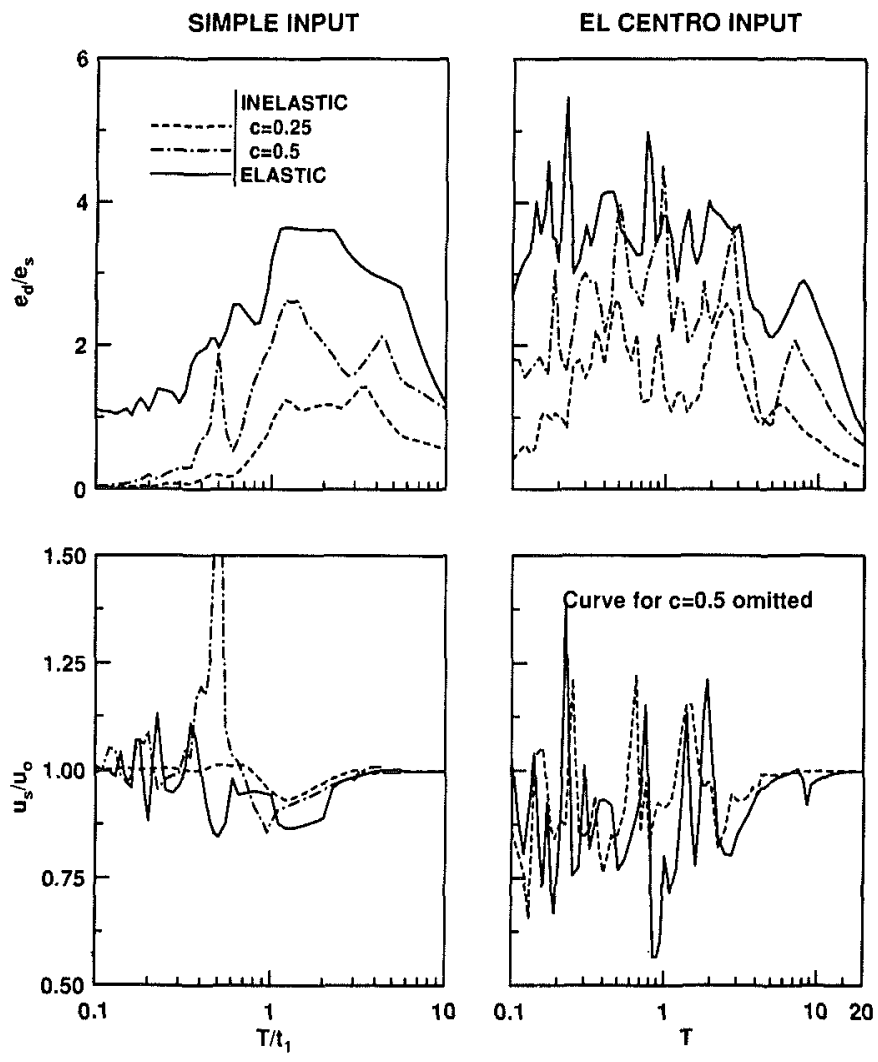

FIG. 7. Ratio of Peak Lateral Deformations of Asymmetric- and Symmetric-Plan Systems $u_{s} / u_{o}$ and Ratio of Dynamic and Static Eccentriclties $e_{d} / e_{s}$ for Elastlc Systems and Inelastlc Systems $\left(e_{p}=e_{s} ; c=0.25\right.$ and 0.5$) ; e_{s} / r=0.2, \Omega_{\theta}=1$, and $\xi$ $=\mathbf{5} \%$

deformation is largest for elastic systems with the smallest $e_{s} / r$ (Fig. 4) but is smaller for inelastic systems (Figs. 5 and 6). As the yield factor $c$ decreases, implying reduction in yield strength and increasing inelastic action, the $e_{d} / e_{s}$ value becomes smaller (Figs. 7 and 8 ). The dynamic eccentricity is generally larger in the case of the El Centro excitation as compared to the simple input.

The lateral deformation $u_{s}$ of velocity-sensitive, asymmetric-plan systems can be significantly different-larger or smaller-than $u_{o}$ of the symmetricplan system (Figs. 4-6). With increasing inelastic action (decreasing $c$ ), this difference tends to decrease, and the deformation of the asymmetric-plan system becomes closer to that of the symmetric-plan system (Figs. 7 and 8).

The ratio $u_{s} / u_{o}$ of lateral deformations of the asymmetric-plan and the corresponding symmetric-plan systems is affected very little by plan asymmetry (Figs. 4-6) or by inelastic behavior (Figs. 7 and 8) in the short-period, acceleration-sensitive and long-period, displacement-sensitive spectral regions of both excitations. In the limit, as $T$ becomes very short or very long, 

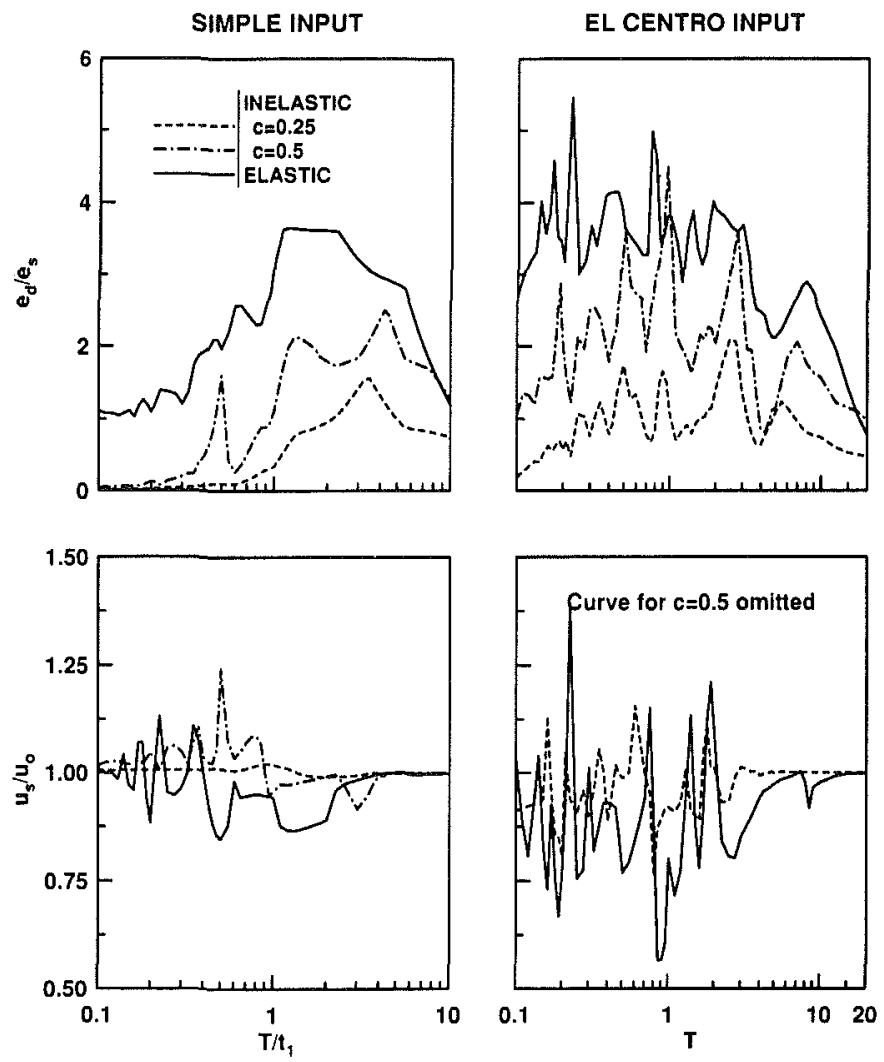

FIG. B. Ratio of Peak Lateral Deformations of Asymmetric- and Symmetric-Plan Systems $u_{s} / u_{o}$ and Ratio of Dynamic and Static Eccentricltles $e_{d} / e_{s}$ for Elastic Systems and Inelastic Systems $\left(e_{p}=0 ; c=0.25\right.$ and 0.5$) ; e_{s} / r=0.2, \Omega_{0}=1$, and $\xi$ $=5 \%$

it can be shown analytically that the lateral deformation of an asymmetricplan system is the same as that of the corresponding symmetric-plan system (Goel and Chopra 1990a). From earlier studies on SDF systems, it is known that inelastic behavior has a smaller influence on the response of long-period, displacement-sensitive systems. Because plan asymmetry also has little influence on the response of such systems, the ratio $u_{s} / u_{o}$ for inelastic systems also approaches one regardless of the yield strength (Figs. 5-8). However, it is not entirely clear why the ratio $u_{s} / u_{o}$ for short-period, accelerationsensitive, inelastic systems also becomes close to one.

The dynamic amplification of torsional deformation, characterized by $e_{d} / e_{s}$, in the acceleration-sensitive spectral region is quite different between elastic and inelastic systems. As $T$ becomes very short, $e_{d} / e_{s}$ for elastic systems approaches one (Fig. 4), indicating that the torsional deformation is equal to that resulting from static application of the lateral force (or base shear) $V_{o}$ at a distance $e_{s}$ from the CS (Goel and Chopra 1990a). However, $e_{d} / e_{s}$ for inelastic systems tends to zero as the period becomes very short, 

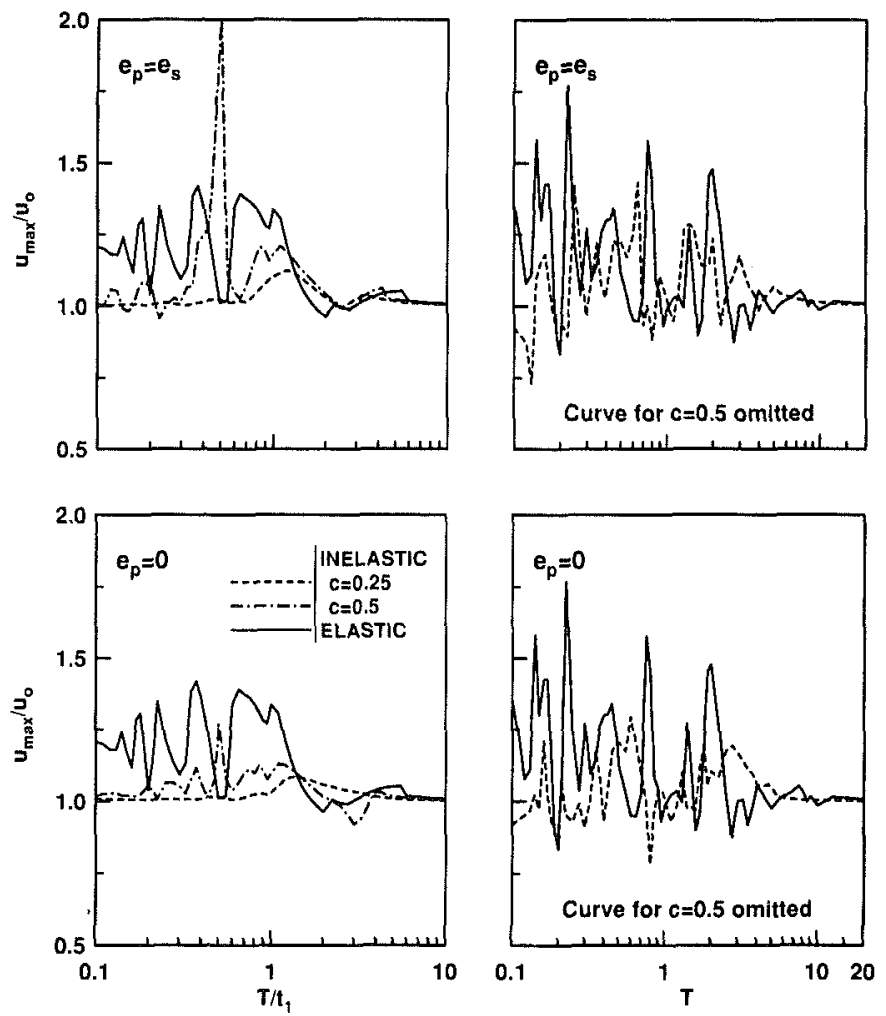

FiG. 9. Ratio of Peak Element Deformatlons of Asymmetric- and Symmetric-Plan Systems $u_{\max } / u_{o}$ for Elastic Systems and Inelastlc Systems $\left(e_{p}=0\right.$ and $e_{s} ; c=0.25$ and 0.5$) ; e_{s} / r=0.2, \Omega_{\mathrm{\theta}}=1$, and $\xi=5 \%$

implying very little torsional deformation (Figs. 5-8). As seen in Fig. 5, there are exceptions to this trend because the torsional stiffness of systems with large stiffness eccentricity and small yield strength may become zero for extended time durations, leading to increased torsional deformation (Goel and Chopra 1990a).

The values of $e_{d} / e_{s}$ in the long-period, displacement-sensitive spectral region tend to be smaller for inelastic systems compared to elastic systems (Figs. 7 and 8 ). In the limit, as $T$ becomes very long, $e_{d} / e_{s}$ for elastic systems can be shown to approach zero, implying very little torsional deformation (Goel and Chopra 1990a). It is not known whether this limiting value of $e_{d} / e_{s}$ is also valid for inelastic systems, but $e_{d} / e_{s}$ for such systems decreases as $T$ becomes long (Figs. 5-8).

The aforementioned observations on the effects of plan asymmetry on the earthquake response of one-story systems, and how these effects are influenced by inelastic action, are more easily discernible from the response results for the various spectral regions of the simple input. They also apply in a rough overall sense to the corresponding spectral regions of the El Cen- 
tro excitation, although the trends are much more irregular and complicated. They would tend to smooth out if the responses were averaged over several earthquake excitations.

The normalized element deformation $u_{\max } / u_{o}$ is plotted against the vibration period $T$ in Fig. 9 for the simple and El Centro excitations. Over a wide range of $T$-values in the acceleration- and velocity-sensitive spectral regions of both excitations, but for a few exceptions, the element deformation is increased by asymmetry of plan. In the displacement-sensitive region, the element deformation is affected very little by plan asymmetry (Fig. 9). The increase in element deformation due to plan asymmetry is generally smaller for inelastic systems, especially for strength-symmetric $\left(e_{p}=0\right)$ systems, compared to elastic systems. With increasing inelastic action, i.e., decreasing $c, u_{\max } / u_{o}$ tends to become closer to one, which implies that, with some exceptions, the element deformation is affected less by plan asymmetry (Fig. 9). These effects of inelastic behavior are more pronounced in the acceleration- and velocity-sensitive regions of the spectrum but are negligible in the displacement-sensitive spectral region. The increase in element deformation of elastic systems due to plan asymmetry is about the same for the two excitations, but for inelastic systems, the increase is larger in the case of the El Centro excitation.

\section{Influence of Frequency Ratio and Stiffness Eccentricity}

The normalized responses $u_{s} / u_{o}$ and $e_{d} / e_{s}$ of elastic systems are plotted in Fig. 10 against the uncoupled torsional-to-lateral frequency ratio $\Omega_{\theta}$ for three values of the normalized eccentricity $e_{s} / r$, and in Fig. 11 against $e_{s} / r$ for several values of $\Omega_{\theta}$. Next, the same responses of inelastic systems with $e_{p}$ $=e_{s}$, and strength-symmetric $\left(e_{p}=0\right)$ inelastic systems, each for two values of $c=0.25$ and 0.5 , are compared with the elastic system responses in Figs. 12-15. Subsequently, normalized element deformation $u_{\max } / u_{o}$ of these inelastic systems and elastic systems are compared in Figs. 16 and 17. Since the effects of plan asymmetry were shown earlier to be most pronounced in the medium-period, velocity-sensitive spectral region, a lateral vibration period value representative of this region is chosen: $T / t_{1}=1.5$ with the simple input and $T=1$ in case of the El Centro input.

For elastic systems, the lateral deformation $u_{s}$ of the asymmetric-plan system is smaller than the deformation $u_{o}$ of the corresponding symmetric-plan system, and over a wide range of parameters, $e_{d} / e_{s}$ exceeds one, indicating dynamic amplification of torsional deformation (Figs. 10 and 11). These effects of plan asymmetry tend to increase as $e_{s} / r$ increases, i.e., the system plan becomes increasingly asymmetric. Thus, $u_{s}$ is increasingly reduced below $u_{o}$ as $e_{s} / r$ increases, and the torsional deformation, as indicated by $e_{d}$, increases as $e_{s} / r$ becomes larger, although the ratio $e_{d} / e_{s}$ is largest for the smallest $e_{s} / r$.

The effects of plan asymmetry on the response of elastic systems depend in an important way on $\Omega_{\theta}$, the ratio of uncoupled torsional and lateral frequencies (Fig. 10). For slightly asymmetric systems (small $e_{s} / r$ ), these effects are most pronounced in systems with equal torsional and lateral frequencies $\left(\Omega_{\theta}=1\right)$ compared to any other value of $\Omega_{\theta}$. As $e_{s} / r$ increases, the asymmetry effects are not necessarily most pronounced at $\Omega_{\theta}=1$. In the case of simple input, $u_{s} / u_{o}$ reaches its minimum value at $\Omega_{\theta}$-values below unity, while $e_{d} / e_{s}$ reaches its maximum at $\Omega_{\theta}$ larger than unity; the trends are not as systematic in the case of the El Centro excitation. In both cases, 

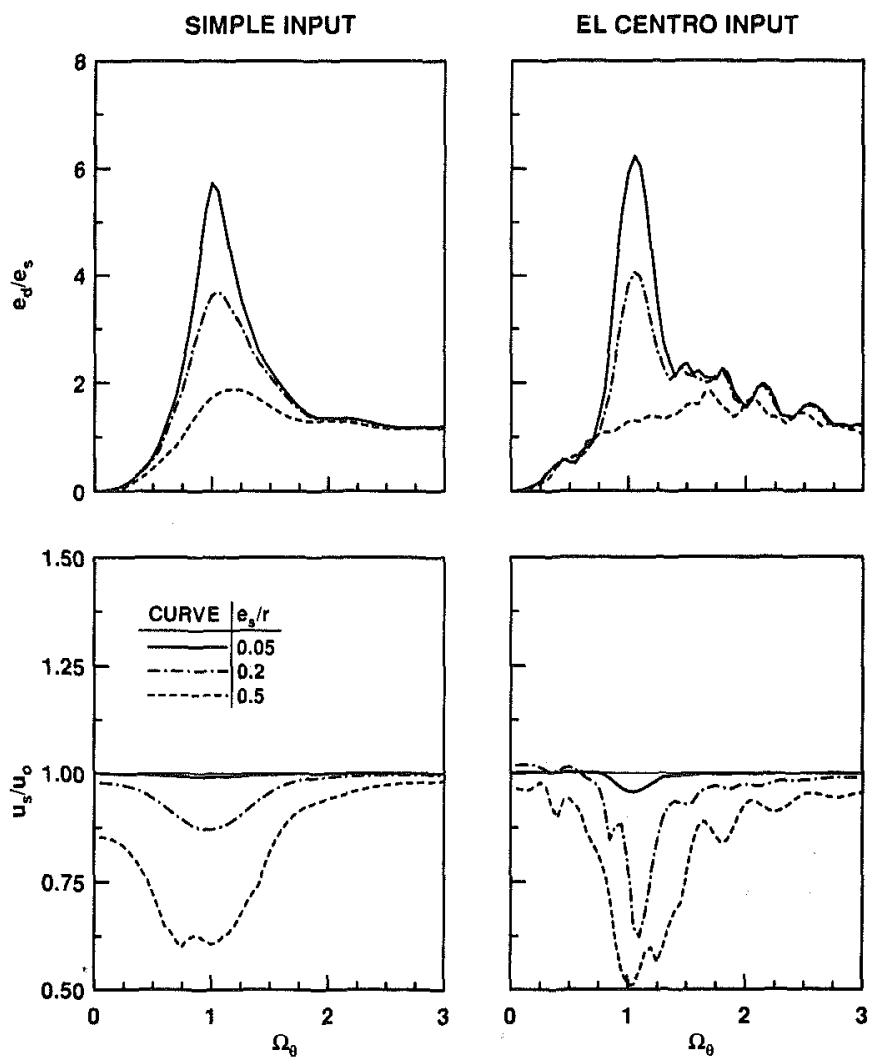

FIG. 10. Ratio of Peak Lateral Deformations of Asymmetrlc- and Symmetric-Plan Systems $u_{s} / u_{o}$ and Ratlo of Dynamlc and Statlc Eccentriclties $e_{d} / e_{s}$ for Elastlc Systems; $T / t_{1}=1.5$ for Simple Input and $T=1$ for El Centro Input; $e_{s} / r=0.05,0.2$, and $0.5 ; \xi=5 \%$

the sharp peak at $\Omega_{\theta}$ around one for systems with small $e_{s} / r$ becomes flatter as $e_{s} / r$ increases, resulting in less dependence of response on $\Omega_{\theta}$ in the range $0.8-1.25$, which covers many buildings (Hart et al. 1975).

The response of inelastic systems to the simple input is affected by $\Omega_{\theta}$ and $e_{s} / r$ in a manner similar to elastic systems but generally to a lesser degree (Figs. 12-15). With decreasing yield factor $c$, which implies increased inelastic action, the peak of $e_{d} / e_{s}$, which still occurs for systems with $\Omega_{\theta}$ around one, becomes smaller and flatter, implying less dynamic amplification of torsional deformation and its decreasing dependence on $\boldsymbol{\Omega}_{\theta}$ (Figs. 12 and 13). Yielding of the system decreases the dynamic amplification of torsional deformation and its dependence on $\Omega_{\theta}$ for two reasons: (1) The uncoupled torsional and lateral vibration frequencies, which are close to each other in a system with initial elastic value of $\Omega_{\theta} \approx 1$, are temporarily separated because of inelastic action; and (2) the system behaves as rigid in torsion for extended time durations as the yield strength decreases (Goel and Chopra 1990a). Secondly, as the yield factor $c$ decreases, implying greater 

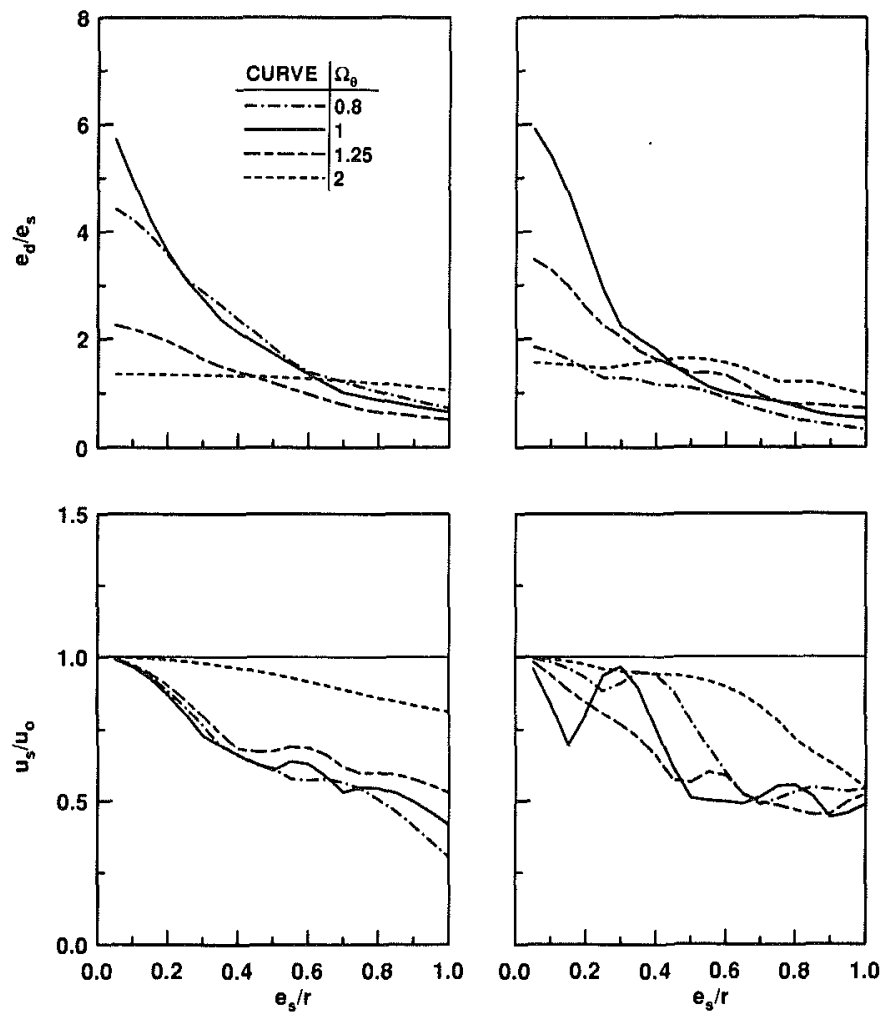

FIG. 11. Ratlo of Peak Lateral Deformations of Asymmetric- and Symmetric-Plan Systems $u_{s} / u_{o}$ and Ratlo of Dynamlc and Statlc Eccentricities $e_{d} / e_{s}$ for Elastlc Systems; $T / t_{1}=1.5$ for Simple Input and $T=1$ for El Centro Input; $\Omega_{\theta}=0.8,1,1.25$, and $2 ; \xi=5 \%$

yielding, $e_{d} / e_{s}$ becomes increasingly independent of $e_{s} / r$ (Figs. 14 and 15) because in a yielding system, the instantaneous CS may move farther from its initial elastic location or shift to the opposite side, leading to cancellation of the effects of eccentricity. It is apparent from Figs. 14 and 15 that inelastic action causes the greatest reduction in $e_{d} / e_{s}$ for systems with the smallest eccentricity ratio, in which case the elastic response is magnified most; the response of systems with large $e_{s} / r$ is reduced to a lesser degree by yielding. For a wide range of $e_{s} / r$ and $\Omega_{\theta}$ values, $e_{d} / e_{s}$ is less than one for system with small yield strength, especially for strength-symmetric systems (Figs. 12-15).

The lateral deformation $u_{s}$ of inelastic systems with $e_{p}=e_{s}$ due to the simple input decreases below $u_{o}$ because of plan asymmetry (Fig. 12), as in the case of elastic systems (Fig. 10). The reduction tends to be the largest for the $\Omega_{\theta}$-value where the $e_{d} / e_{s}$ is the largest. As the yield factor decreases, implying increased inelastic action, the reduction in the lateral deformation due to plan asymmetry becomes smaller in systems with smaller $e_{s} / r$; how- 

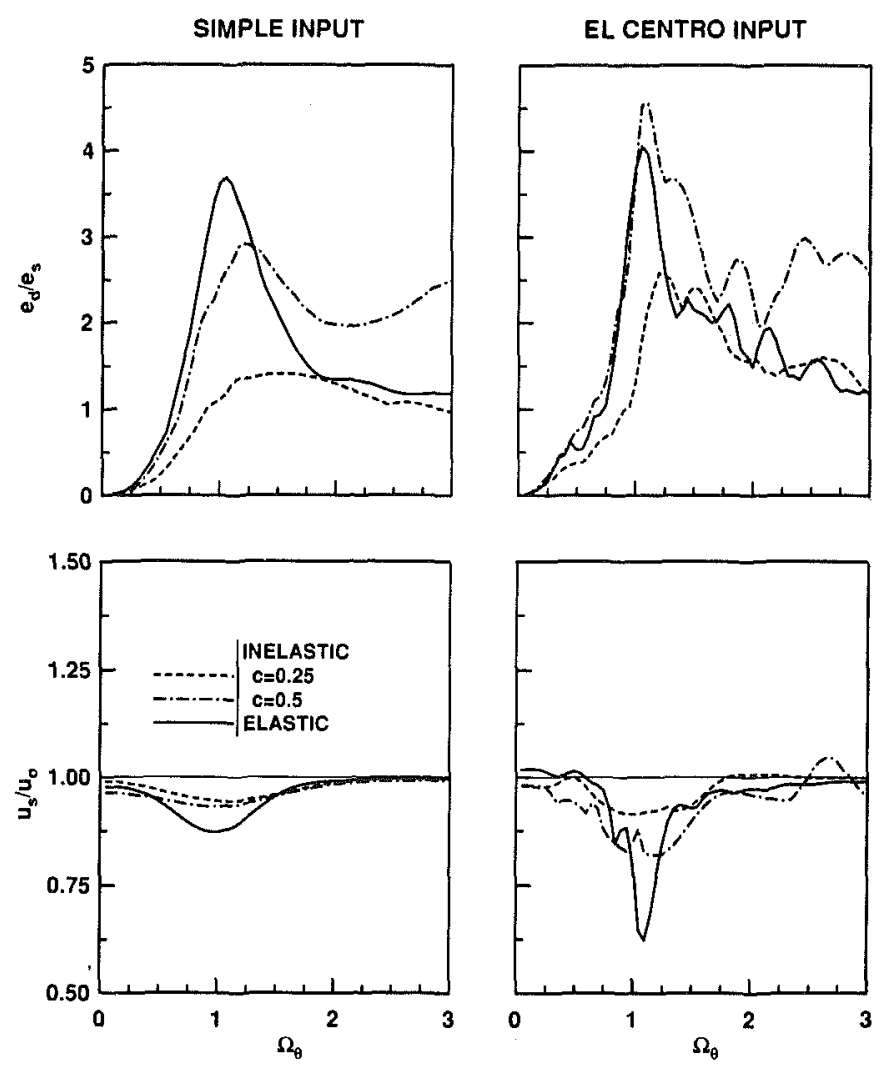

FIG. 12. Ratio of Peak Lateral Deformations of Asymmetric- and Symmetric-Plan Systems $u_{s} / u_{o}$ and Ratio of Dynamic and Static Eccentricities $e_{d} / e_{s}$ for Elastic Systems and Inelastlc Systems $\left(e_{p}=e_{s} ; c=0.25\right.$ and 0.5$) ; T / t_{1}=1.5$ for Simple Input and $T=1$ for El Centro Input; $e_{s} / r=0.2$ and $\xi=5 \%$

ever, the greater reduction occurs for the larger stiffness eccentricities (Fig. 14). In the case of strength-symmetric $\left(e_{p}=0\right)$ inelastic systems, yielding affects the variation of $u_{s} / u_{o}$ with $\Omega_{\theta}$ in a much different way than in inelastic systems with $e_{p}=e_{s}$, resulting in increased lateral deformation for asymmetric-plan systems with larger $\Omega_{\theta}$ and $e_{s} / r$ or very small $\Omega_{\theta}$ (Fig. 13).

As $\Omega_{\theta}$ increases above one, i.e., as the system becomes increasingly stiff in torsion, the normalized responses of the elastic system are less sensitive to $e_{s} / r, u_{s} / u_{o}$ approaches one, indicating that the lateral deformation is affected very little by plan asymmetry, and $e_{d} / e_{s}$ also tends to one, implying that the dynamic torsional deformation is the same as the static torsional deformation defined previously (Fig. 10). These limiting values are analytically demonstrated by Goel and Chopra (1990a). In particular, for systems with $\Omega_{\theta}>2$, the normalized responses are not sensitive to $e_{s} / r$, and the effects of plan asymmetry on lateral deformation may be ignored and the dynamic amplification of torsional deformation neglected. Even for yielding systems, the effects of plan asymmetry on lateral deformation may be ig- 

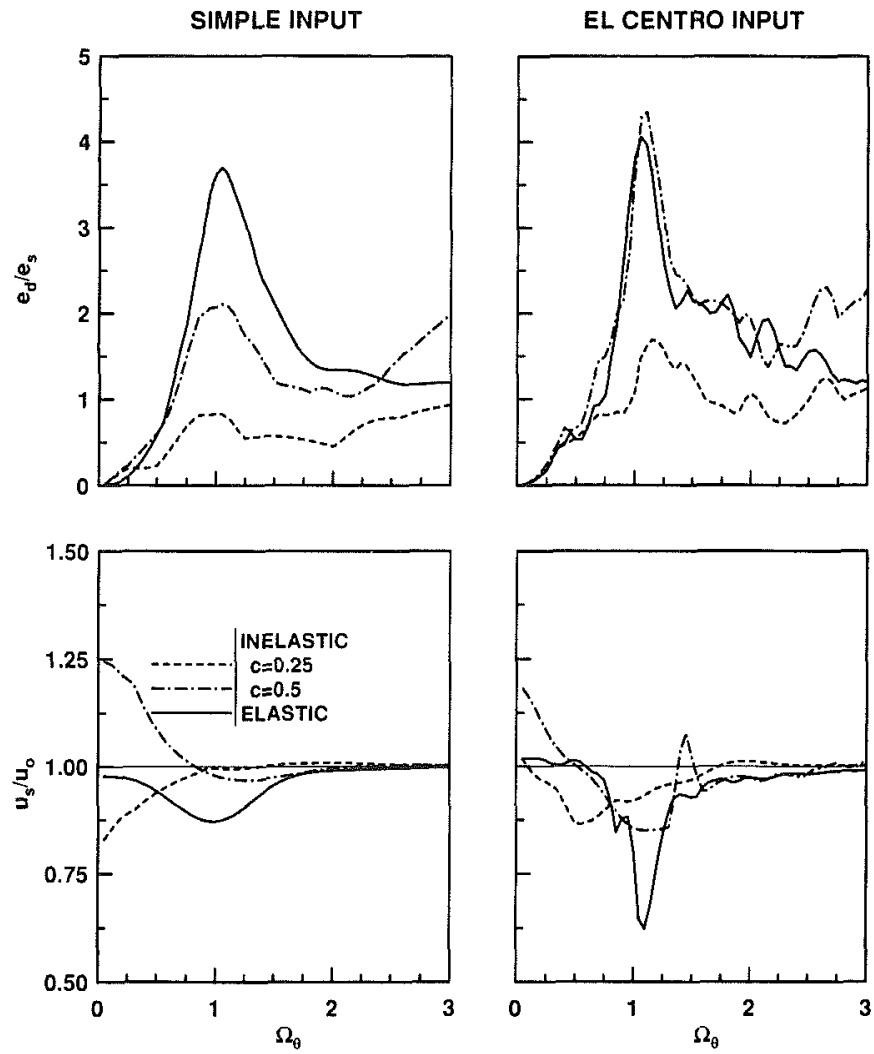

FIG. 13. Ratio of Peak Lateral Deformations of Asymmetric- and Symmetric-Plan Systems $u_{s} / u_{o}$ and Ratio of Dynamic and Static Eccentricities $e_{d} / e_{s}$ for Elastic Systems and Inelastic Systems $\left(e_{p}=0 ; c=0.25\right.$ and 0.5$) ; T / t_{1}=1.5$ for SImple Input and $T=1$ for El Centro Input; $e_{s} / r=0.2$ and $\xi=5 \%$

nored (Figs. 12 and 13). However, the dynamic amplification of torsional deformation may be significant for some values of the yield factor $c$.

As $\Omega_{\theta}$ becomes small, i.e., the elastic system becomes increasingly flexible in torsion, $e_{d}$ approaches zero, implying no torsional deformation, regardless of the stiffness eccentricity. However, the lateral deformation $u_{s}$ is sensitive to the stiffness eccentricity, with a limiting value of $u_{s} / u_{o}$ approximately equal to $1 /\left[1+\left(e_{s} / r\right)^{2}\right]$, which approaches one as $e_{s} / r$ becomes small (Fig. 10). This approximation deteriorates as the stiffness eccentricity increases. These limiting values are analytically demonstrated by Goel and Chopra (1990a). Inelastic action has little influence on $e_{d} / e_{s}$, which tends to zero as $\Omega_{\theta}$ becomes small for all values of $c$ (Figs. 12 and 13). However, the limiting value of $u_{s} / u_{o}$ seems to be different for the two types of inelastic systems and depends on $c$ with no apparently systematic trends.

The reduction in the lateral deformation $u_{s}$ of the asymmetric-plan system below the deformation $u_{o}$ of the corresponding symmetric-plan system due to plan asymmetry, observed in this section, for systems with fixed value 
SIMPLE INPUT
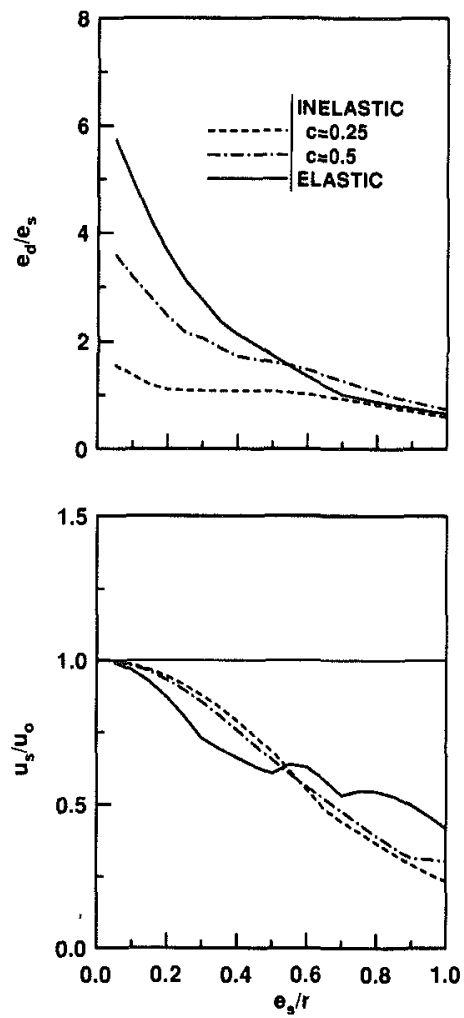

EL CENTRO INPUT
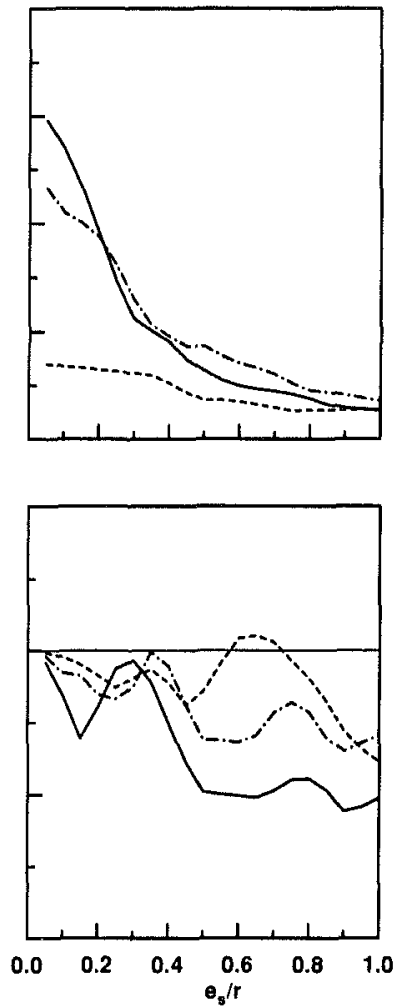

FIG. 14. Ratio of Peak Lateral Deformatlons of Asymmetric- and Symmetric-Plan Systems $u_{s} / u_{o}$ and Ratio of Dynamic and Static Eccentricities $e_{d} / e_{s}$ for Elastlc Systems and Inelastlc Systems $\left(e_{p}=e_{s} ; c=0.25\right.$ and 0.5$) ; T / t_{1}=1.5$ for Simple Input and $T=1$ for El Centro Input; $\Omega_{0}=1$ and $\xi=5 \%$

of the vibration period $T$ may not occur for other values of $T$; on the contrary, as indicated in the preceding section, plan asymmetry may increase $u_{s}$ over $u_{o}$ for systems with other $T$-values. However, the effect of plan asymmetry on the lateral deformation, which makes $u_{s}$ different-larger or smallerthan $u_{o}$ is likely to decrease, i.e., $u_{s}$ is likely to become close to $u_{o}$, with all the factors identified in this section.

The effects of plan asymmetry on the response of inelastic systems are similar in a rough overall sense for the simple input and the El Centro excitation but differ considerably in detail and for certain values of $\Omega_{\theta}$ and $e_{s} / r$. Furthermore, the variation of $u_{s} / u_{o}$ with $\Omega_{\theta}$ or $e_{s} / r$ is much more complicated and irregular for the El Centro excitation. In particular, these complications result in increased lateral deformations in highly asymmetric-plan systems (large $e_{s} / r$ ) for some values of $\Omega_{\theta}$; the increases are relatively small, however. These differences are in part because of the irregular shape of the response spectrum for a single ground motion. They are likely to decrease if the results were averaged over several ground motions. The values 
SIMPLE INPUT
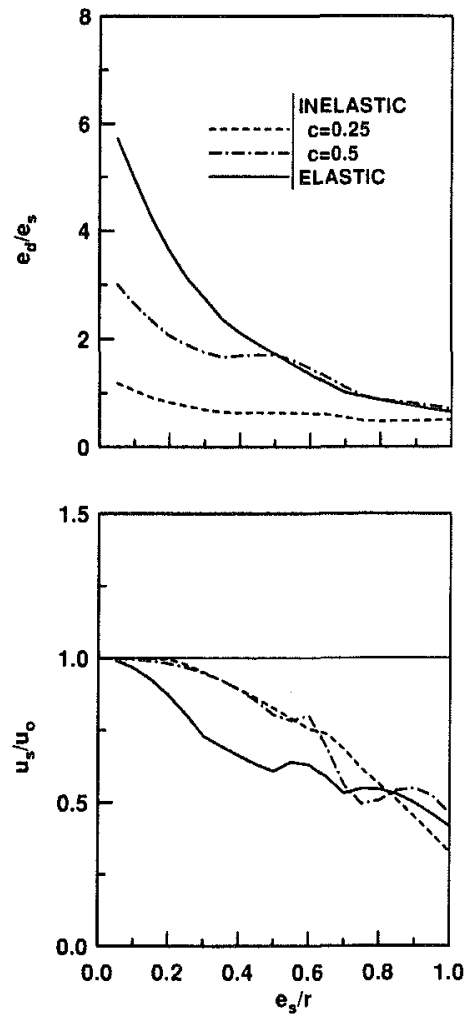

EL CENTRO INPUT
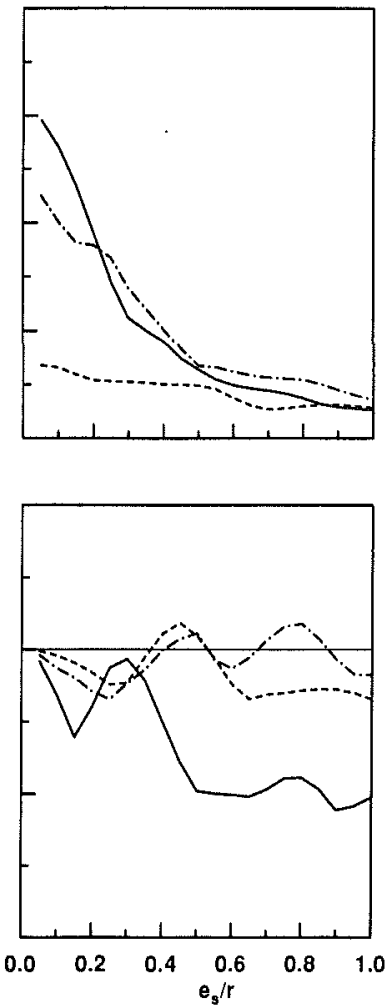

FIG. 15. Ratio of Peak Lateral Deformations of Asymmetric- and Symmetric-Plan Systems $u_{s} / u_{o}$ and Ratio of Dynamic and Static Eccentricities $e_{d} / e_{s}$ for Elastic Systems and Inelastic Systems $\left(e_{p}=0 ; c=0.25\right.$ and 0.5$) ; T / t_{1}=1.5$ for Simple Input and $T=1$ for El Centro Input; $\Omega_{\theta}=1$ and $\xi=5 \%$

of $e_{d} / e_{s}$ for elastic systems are about the same for both excitations, but in the case of inelastic systems, they tend to be larger for the El Centro input.

Over a wide range of $\Omega_{\theta}$ and $e_{s} / r$ values, the maximum element deformation $u_{\max }$ in an asymmetric-plan system due to the simple input is generally, but not always, larger than the element deformation $u_{o}$ in the corresponding symmetric-plan system (Figs. 16 and 17). This increase in $u_{\max }$ tends to increase with the stiffness eccentricity $e_{s} / r$ (Fig. 17), but its dependence on $\Omega_{0}$ is not strong or systematic (Fig. 16). The increase in $u_{\max }$ due to plan asymmetry may be larger or smaller in inelastic systems. For the smaller values of $c$, implying much yielding, $u_{\max } / u_{o}$ is close to one (Fig. $16)$, especially for strength-symmetric $\left(e_{p}=0\right)$ systems. However, for some values of $c, u_{\max } / u_{o}$ for inelastic systems may be larger than that for elastic systems (Fig. 16). As an exception to the general trend, the element deformation decreases because of plan asymmetry for very small values of $\Omega_{\theta}$ in the case of simple input. Furthermore, as the yield factor decreases, implying increased inelastic action, $u_{\max } / u_{o}$ becomes increasingly insensitive to $\Omega_{\theta}$ (Fig. 
SIMPLE INPUT
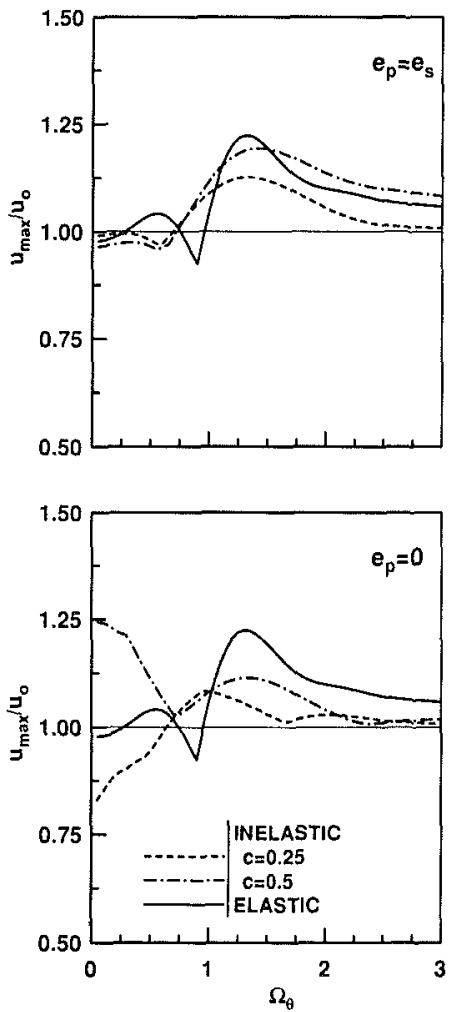

EL CENTRO INPUT
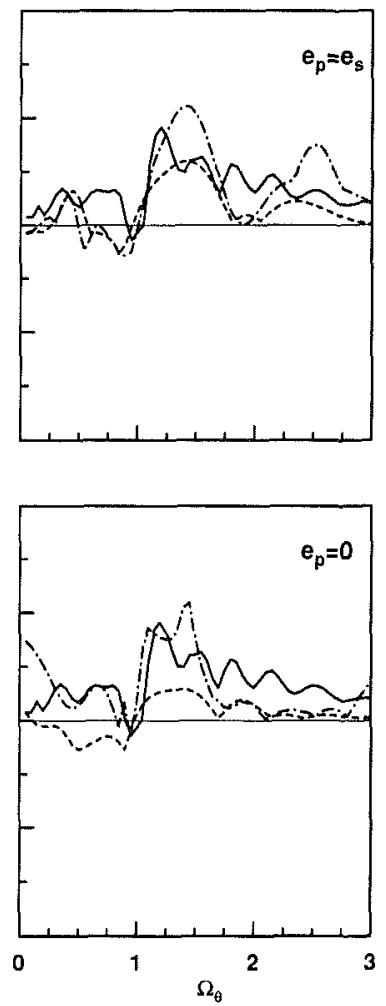

FIG. 16. Ratlo of Peak Element Deformations of Asymmetric- and SymmetricPlan Systems $u_{\max } / u_{o}$ for Elastic Systems and Inelastic Systems ( $e_{p}=0$ and $e_{s} ; c$ $=0.25$ and 0.5$) ; T / t_{1}=1.5$ for Simple Input and $T=1$ for El Centro Input; $e_{s} / r=$ 0.2 and $\xi=5 \%$

16). While the variation of $u_{\max } / u_{o}$ with $\Omega_{\theta}$ or $e_{s} / r$ is gradual in case of the simple input, it is irregular for the El Centro excitation. In particular, the $u_{\max }$ increases over $u_{o}$, as in the case of simple input, for some values of $e_{s} / r$, but decreases relative to $u_{o}$ for other values of $e_{s} / r$ (Fig. 17). Similarly $u_{\max }$ decreases below $u_{o}$ because of plan asymmetry for sporadic values of $\Omega_{\theta}$ (Fig. 16). If the results were averaged over several ground motions, the variations would tend to be smoother.

\section{Conclusions}

Plan asymmetry causes torsional deformation, which does not occur in the corresponding symmetric-plan system; modifies the lateral deformation experienced by the corresponding symmetric-plan system, resulting in a smaller or larger deformation; and generally increases the largest of peak deformations among all resisting elements compared to the deformation of the same element in the corresponding symmetric-plan system.

The effects of plan asymmetry on the lateral and torsional deformations 

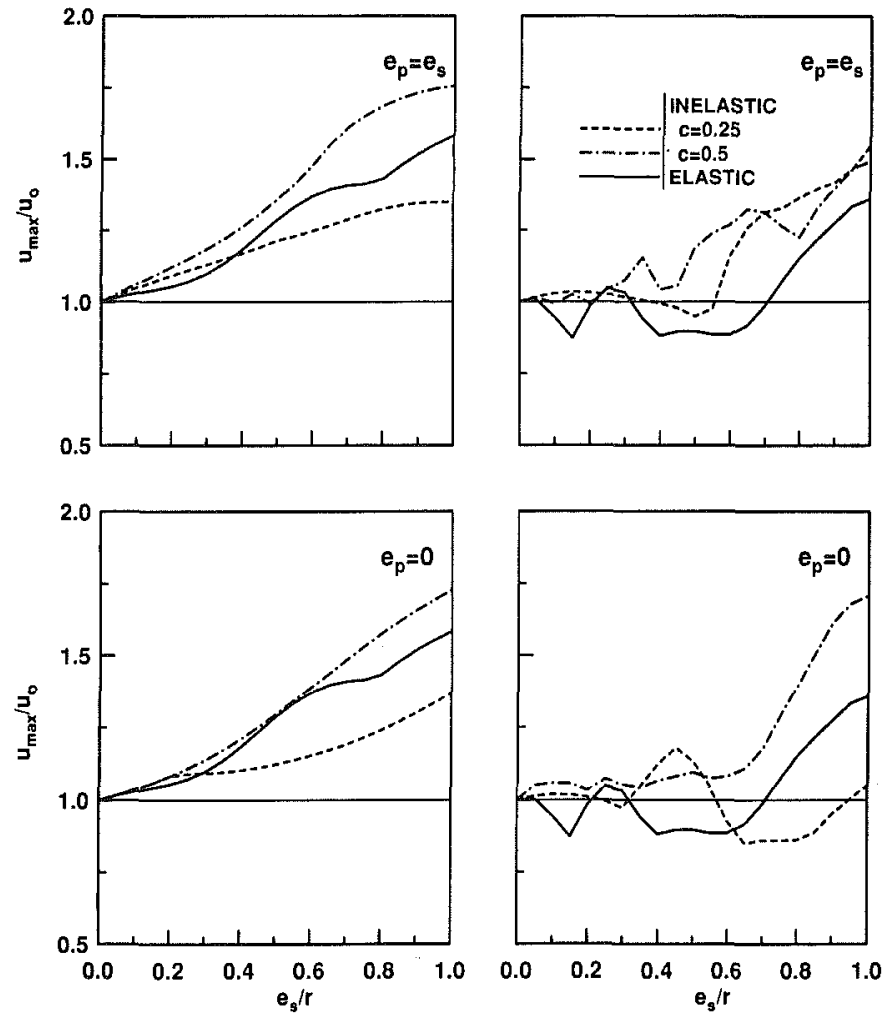

FIG. 17. Ratio of Peak Element Deformations of Asymmetric- and SymmetrlcPlan Systems $u_{\max } / u_{o}$ for Elastic Systems and Inelastic Systems $\left(e_{p}=0\right.$ and $e_{s} ; c$ $=0.25$ and 0.5$) ; T / t_{1}=1.5$ for SImple Input and $T=1$ for El Centro Input; $\Omega_{\theta}=$ 1 and $\xi=5 \%$

of elastic systems depend significantly on the uncoupled lateral vibration period of the system, especially for realistic excitations, such as the El Centro ground motion, and are most pronounced in the medium-period, velocitysensitive spectral region and the neighboring transition regions of the spectrum. However, the period dependence of the effects of plan asymmetry is less pronounced for inelastic systems and decreases with increased yielding that results from decreasing yield strength.

The lateral deformation of short-period, acceleration-sensitive and longperiod, displacement-sensitive systems is affected very little by plan asymmetry or by inelastic behavior. The dynamic amplification of torsional deformation is quite different between elastic and inelastic systems. The dynamic torsional deformation of elastic systems becomes equal to its static value - the deformation due to $V_{o}$ applied at a distance $e_{s}$ - for very shortperiod systems, and zero for very long-period systems. However, very longperiod as well as very short-period inelastic systems generally experience very little torsional deformation. 
The effects of plan asymmetry on the response of elastic systems depend in an important way on the ratio $\Omega_{\theta}$ of uncoupled torsional and lateral frequencies, and are most pronounced in systems with close frequencies. In particular, considerable dynamic amplification of the torsional deformation occurs for systems with $\Omega_{\theta}$ around unity, and this amplification is greater for slightly asymmetric (small values of normalized stiffness eccentricity $\left.e_{s} / r\right)$ systems. The modification of lateral deformation is largest for highly asymmetric (large $e_{s} / r$ ) systems. Because of yielding of the system, the peak of $e_{d} / e_{s}$, which still occurs for systems with $\Omega_{\theta}$ around one, becomes smaller and flatter implying less dynamic amplification of torsional deformation and its decreasing dependence on $\Omega_{\theta}$. As the yield strength of the system decreases, implying increased yielding, the torsional deformation decreases and it becomes increasingly insensitive to $e_{s} / r$.

The lateral deformation of torsionally very stiff (large $\Omega_{\theta}$ ) systems is affected very little by plan asymmetry, and their dynamic torsional deformation is essentially the same as the static value. In particular, for systems with $\Omega_{\theta}>2$, the normalized responses are not sensitive to $e_{s} / r$, and the effects of plan asymmetry on lateral deformation may be ignored and the dynamic amplification of torsional deformation neglected. This conclusion is valid for elastic systems and generally for inelastic systems as well, except that in the latter case the dynamic amplification of torsional deformation may be significant for some values of yield strength.

The largest of the peak deformations among all resisting elements is generally increased by plan asymmetry for systems in the acceleration- and velocity-sensitive regions of the spectrum; however, the element deformation is affected little by plan asymmetry in the displacement-sensitive region. For elastic systems, this increase in element deformation becomes larger as the stiffness eccentricity increases and is relatively insensitive to the frequency ratio. The increase in element deformation due to plan asymmetry is generally smaller for inelastic systems, especially for strength-symmetric $\left(e_{p}=\right.$ 0 ) systems, compared to elastic systems. With increasing inelastic action, the element deformation in an asymmetric-plan system becomes closer to that of the symmetric-plan system.

As mentioned earlier, the response of inelastic systems is affected less by plan asymmetry compared to elastic systems. Between the two types of inelastic systems considered, the response of strength-symmetric systems is affected by plan asymmetry to a generally smaller degree compared to systems with equal stiffness and strength eccentricities. In particular, the dynamic amplification of torsional deformation is smaller, and the increase in element deformation due to plan asymmetry is less in strength-symmetric systems.

The effects of plan asymmetry on structural response are similar in an overall sense for the corresponding spectral regions of the simple input and the El Centro excitation, but may differ considerably in detail; furthermore, the variation of these effects with various systems parameters is more complicated in the case of the latter excitation. These complications are in part due to the irregular shape of the response spectrum for a single ground motion; they would depend on the ground-motion properties and details, and are likely to decrease if the results are averaged over several ground motions. Because the effects of plan asymmetry have been investigated in this paper using a broad-frequency-band earthquake motion, the conclusions may not be valid for narrow-band excitations. 
This research investigation is supported by the National Science Foundation under grant BCS-8921932. The writers are grateful for this support.

\section{Appendix. References}

Bozorgnia, Y., and Tso, W. K. (1986). "Inelastic earthquake response of asymmetric structures." J. Struct. Engrg., ASCE, 112(2), 338-399.

Erdik, M. O. (1975). "Torsional effects in dynamically excited structures," thesis presented to Rice University, at Houston, Tex., in partial fulfillment of the requirements for the degree of Doctor of Philosophy.

Esteva, L. (1987). "Earthquake engineering research and practice in Mexico after the 1985 earthquakes." Bull. New Zealand Nat. Soc. for Earthquake Engrg., 20(3), 159-200.

Goel, R. K., and Chopra, A. K. (1990a). "Inelastic seismic response of one-story, asymmetric-plan systems." Report No. UCB/EERC-90/14, Earthquake Engrg. Res. Ctr., Univ. of California, Berkeley, Calif.

Goel, R. K., and Chopra, A. K. (1990b). "Inelastic seismic response of one-story, asymmetric-plan systems: Effects of stiffness and strength distribution." Earthquake Engrg. Struct. Dyn., 19(7), 949-970.

Hart, G. C., DiJulio, R. M., and Lew, M. (1975). "Torsional response of high-rise buildings." J. Struct. Div., ASCE, 101(2), 397-416.

Hejal, R., and Chopra, A. K. (1989). "Lateral-torsional coupling in earthquake response of frame buildings." J. Struct. Engrg., ASCE, 115(4), 852-867.

Irvine, H. M., and Kountouris, G. E. (1980). "Peak ductility demands in simple torsionally unbalanced building models subjected to earthquake ground excitation." Proc. 7th World Conf. on Earthquake Engrg., International Association of Earthquake Engineering, Istanbul, Turkey, 4, 117-120.

Kan, C. L., and Chopra, A. K. (1981). "Torsional coupling and earthquake response of simple elastic and inelastic systems." J. Struct. Div., ASCE, 107(8), 15691588.

Sadek, A. W., and Tso, W. K. (1988). "Strength eccentricity concept for inelastic analysis of asymmetric structures." Proc. 9th Worth Conf. on Earthquake Engrg., International Association of Earthquake Engineering, Tokyo, Japan, 5, 91-96.

Tso, W. K., and Hongshan, Y. (1990). "Additional seismic inelastic deformation caused by structural asymmetry." Earthquake Engrg. Struct. Dyn., 19(2), 243258.

Tso, W. K., and Sadek, A. W. (1985). "Inelastic seismic response of simple eccentric structures." Earthquake Engrg. Struct. Dyn., 13(2), 255-269.

Veletsos, A. S., and Vann, W. P. (1971). "Response of ground-excited elastoplastic systems." J. Struct. Div., ASCE, 97(4), 1257-1281. 